\title{
Processo de trabalho de Agentes Comunitários e saúde mental: percepções de trabalhadores da saúde de um município do interior do Ceará
}

\author{
Work process of Community Agents and mental health: health workers's perceptions in a \\ municipality in the interior of Ceará \\ Proceso de trabajo de Agentes Comunitarios y salud mental: percepciones de los trabajadores de \\ salud en un municipio del interior de Ceará
}

\author{
Idária Samira da Silva Costa \\ ORCID: https://orcid.org/0000-0003-4290-5281 \\ Escola de Saúde Pública do Ceará, Brasil \\ E-mail: idaria_samira@outlook.com \\ Ana Carolina Souza Torres \\ ORCID: https://orcid.org/0000-0002-8837-4781 \\ Universidade Federal da Bahia, Brasil \\ E-mail: acarolina_st@yahoo.com.br \\ Maria Iracema Capistrano Bezerra \\ ORCID: https://orcid.org/0000-0002-6095-1882 \\ Secretaria da Saúde do Estado do Ceará, Brasil \\ E-mail: iracemabezerra@yahoo.com.br \\ Ronaldo Rodrigues Pires \\ ORCID: https://orcid.org/0000-0003-2217-0097 \\ Universidade Estadual do Ceará, Brasil \\ E-mail: ronaldo.pires.psi@gmail.com
}

\begin{abstract}
Resumo
Introdução: O papel do agente comunitário de saúde na Atenção Primária é fundamental, pois o mesmo é o elo entre a comunidade e a equipe de saúde. No desenvolvimento das atividades laborais estes profissionais enfrentam muitos desafios, podendo gerar frustrações e desesperança, causando sofrimento psíquico. Objetivo: Compreender os fatores que geram sofrimento mental na rotina laboral dos agentes comunitários de saúde. Método: Pesquisa qualitativa, com uso de entrevistas semiestruturadas realizadas entre o mês de agosto e setembro de 2020 com sete agentes comunitárias de saúde atuantes numa Unidade Básica de Saúde de Iguatu- Ceará. O material empírico gerado foi transcrito e analisado pela técnica de Análise de Conteúdo do tipo temática. Resultados: O contexto laboral gera sofrimento psíquico por ser permeado por pressões, cobranças, muitos impasses na microárea, acúmulo de trabalho e pelo desafio de ser moradora, trabalhadora e usuária dos serviços de saúde no mesmo território. Conclusão: É necessário o planejamento de ações para aperfeiçoar o processo de trabalho do agente comunitário de saúde, a fim de reduzir o sofrimento mental e melhorar qualidade de vida dessas trabalhadoras.
\end{abstract}

Palavras-chave: Saúde mental; Saúde do trabalhador; Agentes comunitários de saúde; Atenção Primária à Saúde.

\begin{abstract}
Introduction: The role of the community health agent in Primary Care is fundamental, as it is the link between the community and the health team. In the development of work activities, these professionals face many challenges, which can generate frustration and hopelessness, causing psychological distress. Objective: To understand the factors that generate mental suffering in the work routine of community health agents. Method: Qualitative research, using semi-structured interviews carried out between August and September 2020 with seven community health agents working in a Basic Health Unit in Iguatu- Ceará. The empirical material generated was transcribed and analyzed using thematic Content Analysis technique. Results: The work context generates psychological distress because it is permeated by pressure, demands, many impasses in the micro-area, accumulation of work and the challenge of being a resident, worker and user of health services in the same territory. Conclusion: It is necessary to plan actions to improve the work process of the community health agent, in order to reduce mental suffering and improve the quality of life of these workers.
\end{abstract}

Keywords: Mental health; Occupational health; Community health workers; Primary Health Care. 


\begin{abstract}
Resumen
Introducción: El papel del agente comunitario de salud en Atención Primaria es fundamental, ya que es el vínculo entre la comunidad y el equipo de salud. En el desarrollo de las actividades laborales, estos profesionales enfrentan muchos desafíos, los cuales pueden generar frustración y desesperanza, provocando malestar psicológico. Objetivo: Comprender los factores que generan sufrimiento mental en la rutina laboral de los trabajadores comunitarios de salud. Método: Investigación cualitativa, mediante entrevistas semiestructuradas realizadas entre agosto y septiembre de 2020 con siete agentes comunitarios de salud que laboran en una Unidad Básica de Salud en Iguatu- Ceará. El material empírico generado fue transcrito y analizado mediante la técnica de Análisis de Contenido temático. Resultados: El contexto laboral genera malestar psicológico porque está impregnado de presiones, demandas, muchos impasses en la microzona, acumulación de trabajo y el desafío de ser residente, trabajador y usuario de los servicios de salud en un mismo territorio. Conclusión: Es necesario planificar acciones para mejorar el proceso de trabajo del agente comunitario de salud, con el fin de reducir el sufrimiento mental y mejorar la calidad de vida de estos trabajadores.
\end{abstract}

Palabras clave: Salud mental; Salud laboral; Agentes comunitarios de salud; Atención Primaria de Salud.

\title{
1. Introdução
}

O surgimento do Agente Comunitário de Saúde (ACS) no Brasil se deu na década de 1970 com a finalidade de reduzir a mortalidade materno-infantil, através de orientações sobre a prevenção das doenças por meio de informações sobre cuidados com a saúde. Devido ao êxito no trabalho desses profissionais foi criado o Programa de Agentes Comunitários de Saúde (PACS) em 1991 com o objetivo de desenvolver atividades de prevenção de doenças e promoção da saúde, realizando ações educativas individuais e coletivas nos domicílios e na comunidade. Logo após, em 1994, o PACS foi incorporado ao Programa Saúde da Família (PSF) hoje denominado Estratégia Saúde da Família (ESF) (Speroni, et al., 2016).

O trabalho profissional dos agentes de saúde só foi reconhecido em 2002, através da Lei $\mathrm{N}^{\circ} 10.507$ aprovada pelo Governo Federal. Porém, somente em 2006 o ACS teve suas atividades regulamentadas pela Lei Federal $\mathrm{N}^{\circ} 11.350$, que dispõe sobre suas competências dentro da Atenção Primária, além de considerar sobre a realização do curso introdutório para a formação inicial e o ensino fundamental completo como requisito para o exercício profissional (Brasil, 2002; Brasil, 2006).

O papel do ACS no desenvolvimento do trabalho na Atenção Primária a Saúde (APS) é fundamental, pois o mesmo é o elo entre a comunidade e a equipe de saúde, integrando os usuários e profissionais e facilitando a criação de vínculo e confiança. O fato de viver e trabalhar no território permite que o ACS identifique as dificuldades vivenciadas pelos usuários sendo um agente transformador das situações que estejam afetando a saúde e a qualidade de vida da população (Pereira, et al., 2018).

Ao desenvolver as suas atividades os agentes comunitários de saúde enfrentam muitos desafios que podem gerar frustrações e desesperança na realização de suas atribuições causando sofrimento psíquico. Essas dificuldades vão desde a desvalorização do seu trabalho por parte da comunidade até o sentimento de impotência diante da realidade social, econômica e de saúde-doença vivenciadas pelos moradores, no qual afeta o processo de cuidado pelo qual esse profissional é responsável (Guanaes-Lorenzi \& Pinheiro, 2016).

Ademais, estes profissionais vivenciam situações que oferecem riscos à saúde e ao seu bem-estar, circunstâncias em que falta apoio da gestão e do poder público, relações interpessoais enfraquecidas com os colegas de trabalho, desestrutura da unidade de saúde, sentimento de medo e insegurança diante de algumas situações que se deparam na comunidade e as longas caminhadas em ruas que não oferecem salubridade (Pereira, et al., 2018).

Pesquisas evidenciam índices elevados de sofrimento mental nos profissionais da saúde. No que se refere ao agente comunitário de saúde, este foi escolhido como objeto de estudo, pois a sua atuação e atribuições abrangem todas as particularidades, especificidades do território e, além disso, residir e trabalhar no mesmo espaço pode trazer prejuízos à saúde, principalmente à saúde mental.

O estudo em questão pretende contribuir no meio acadêmico e social, de forma a agregar conhecimentos científicos 
no arcabouço de pesquisas relacionadas à saúde mental dos ACS, por conseguinte ampliar a compreensão sobre suas práticas diante do atual contexto que estão inseridos.

O desenvolvimento dessa pesquisa buscou compreender os fatores que geram sofrimento mental na rotina laboral dos Agentes Comunitários de Saúde, os quais são responsáveis juntamente com a Equipe Saúde da Família pelo cuidado das pessoas adscritas na sua microárea. Dessa forma, o presente artigo pretende contribuir com reflexões, construídas a partir da percepção desses trabalhadores da saúde, as quais fornecerão subsídios para a construção de estratégias voltadas ao cuidado em saúde mental desses profissionais, com intuito de proporcionar melhores condições de trabalho e, consequentemente, qualificar o acolhimento e assistência voltados à população.

\section{Metodologia}

Este estudo caracteriza-se como uma pesquisa de abordagem qualitativa, realizada na cidade de Iguatu, situada na região Centro-Sul do estado do Ceará, a 377 km da capital Fortaleza. Foi realizada com 07 Agentes Comunitários de Saúde da UBS Dr. Ernani Barreira.

A UBS Dr. Ernani Barreira é localizada na zona urbana de Iguatu e é constituída por três equipes de Saúde da Família, foi escolhida como local para execução da pesquisa por ser o território de atuação da equipe de referência residente que compõem a Residência Integrada em Saúde da Escola de Saúde Pública do Ceará (RIS/CE), composta por três profissionais, sendo estes dois enfermeiros e um cirurgião-dentista.

Os participantes foram avaliados quanto aos critérios de inclusão (tempo mínimo 6 meses de trabalho na atual UBS) e exclusão (afastamento das atividades laborais por férias, licença e/ou doença durante a coleta de dados).

A pesquisa desenvolveu-se entre o mês de agosto e setembro de 2020 por meio de entrevistas semiestruturadas, estas foram realizadas em local reservado na UBS pela pesquisadora responsável. Após a coleta dos dados foi realizado a transcrição das falas dos participantes na íntegra. Sendo posteriormente feita a leitura das entrevistas com a finalidade de apreender os sentidos que os participantes deixaram transparecer em suas falas.

Para análise do material empírico utilizou-se a Análise de Conteúdo do tipo temática de Minayo (Silva Filho, 2019). Dessa forma, analisar os dados consistem na descrição, interpretação, compreensão e valorização dos mesmos, pois essa fase possibilita um olhar mais atento para os dados da pesquisa. Ademais, permite a descoberta de códigos sociais a partir das falas, símbolos e observações, assim como articulá-los com a teoria que fundamentou o projeto ou com outras leituras teóricas. Em seguida agrupou-se os elementos, ideias ou expressões com características comuns e que se relacionam entre si para formar as categorias temáticas.

Este estudo seguiu as normatizações éticas instituídas pelas Resoluções № 510 de 07 de abril de 2016 e № 466 de 12 de dezembro de 2012, do Conselho Nacional de Saúde (CNS), que aprova as diretrizes e normas regulamentadoras de pesquisas envolvendo seres humanos, bem como referentes às pesquisas envolvendo seres humanos em Ciências Humanas e Sociais, respectivamente. Assim, os princípios de liberdade, autonomia, confidencialidade e valores culturais, sociais, morais e religiosos foram respeitados por parte do pesquisador (Brasil, 2016; Brasil, 2012).

O projeto de pesquisa foi submetido à aprovação do Comitê de Ética em Pesquisa (CEP), Escola de Saúde Pública do Ceará (ESP-CE) via Plataforma Brasil, e obteve parecer favorável sob o número CAAE: 4.129.268.

\section{Resultados}

Os resultados obtidos no presente estudo serão abordados a seguir em duas etapas, primeiramente apresentando a caracterização das participantes da pesquisa, para posteriormente ressaltar as categorias temáticas construídas a partir das entrevistas. 
Assim, todos os participantes eram do sexo feminino (100\%), com faixa etária predominantemente compreendida entre 33 e 54 anos (57,14\%). Já em relação ao estado civil, as entrevistadas eram solteiras $(57,14 \%)$ e casadas $(42,85 \%)$.

No que tange à renda familiar, tomando como base o valor do salário mínimo no ano de 2020 (R \$1.045,00), a maioria das ACS dispõe de 2 a 3 salários mínimos (71,42\%). Apenas duas ACS possuem renda de 4 a 5 salários mínimos (28,57\%). Quanto à escolaridade, três $(42,85 \%)$ das sete participantes têm ensino superior incompleto, duas $(28,57 \%)$ possuem ensino médio completo, uma $(14,28 \%)$ possui ensino superior completo e outra $(14,28 \%)$ possui ensino fundamental completo.

Com relação ao tempo de atuação como agente comunitária de saúde, cinco participantes $(71,42 \%)$ trabalham há mais de 5 anos, uma ACS (14,28\%) atua por um período de 3 a 5 anos e outra (14,28\%) de 6 meses a 1 ano. Relativo ao tempo de lotação na atual UBS, três ACS (42,85\%) trabalham há mais de 5 anos, duas (28,57\%) estão lotadas entre 3 a 5 anos, uma ACS $(14,28 \%)$ entre 1 a 3 anos e outra $(14,28 \%)$ de 6 meses a 1 ano.

A partir da análise dos dados foram depreendidas duas categorias por meio dos recortes temáticos apresentados no Quadro 1.

Quadro 1 - Categorias temáticas e recortes temáticos das entrevistas.

\begin{tabular}{|c|c|}
\hline Categorias Temáticas & Recortes Temáticos \\
\hline $\begin{array}{l}\text { Interface trabalho e saúde } \\
\text { mental }\end{array}$ & 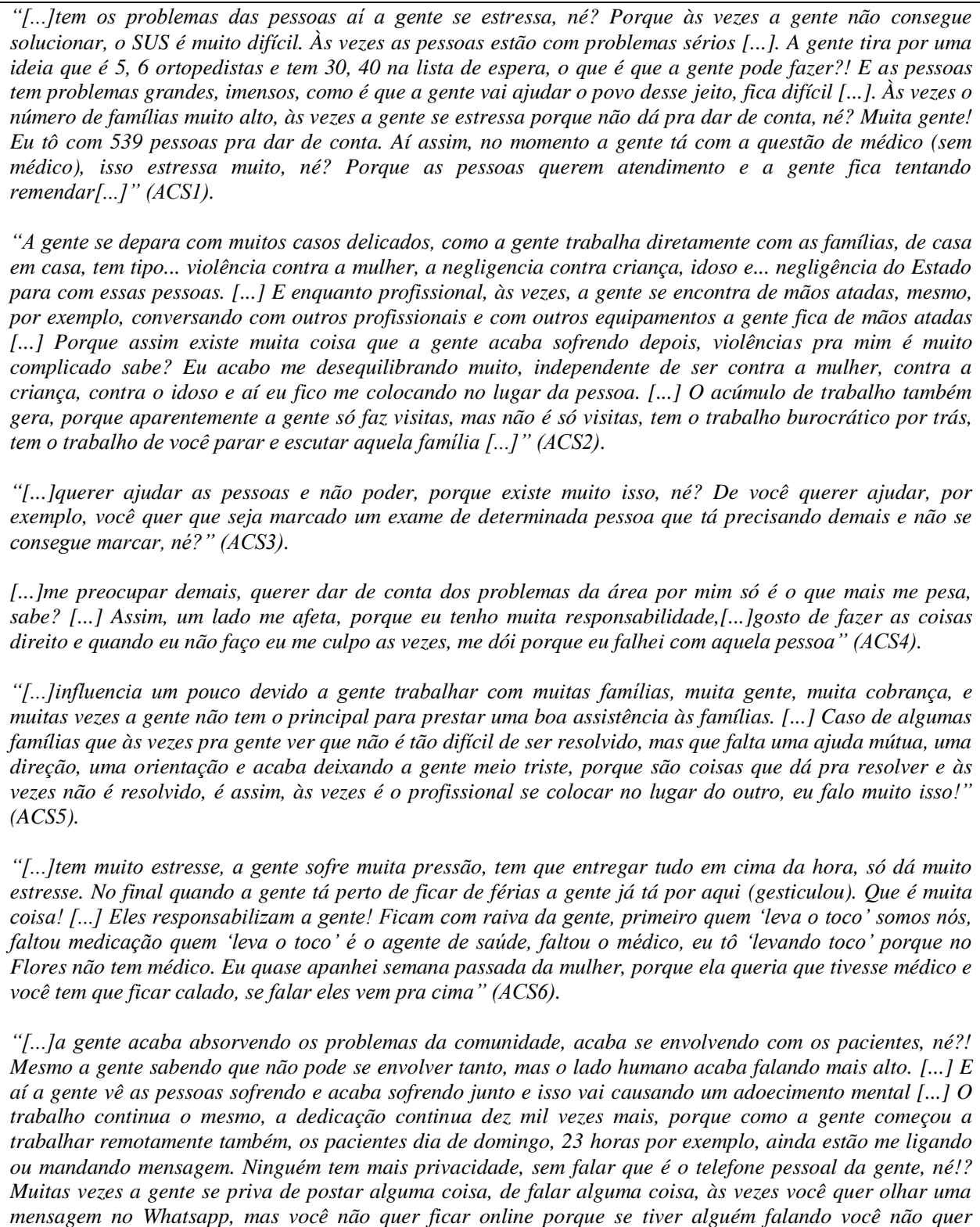 \\
\hline
\end{tabular}




\begin{tabular}{|c|c|}
\hline & esponder" (ACS7) \\
\hline $\begin{array}{l}\text { Estratégias de cuidado em } \\
\text { saúde mental. }\end{array}$ & 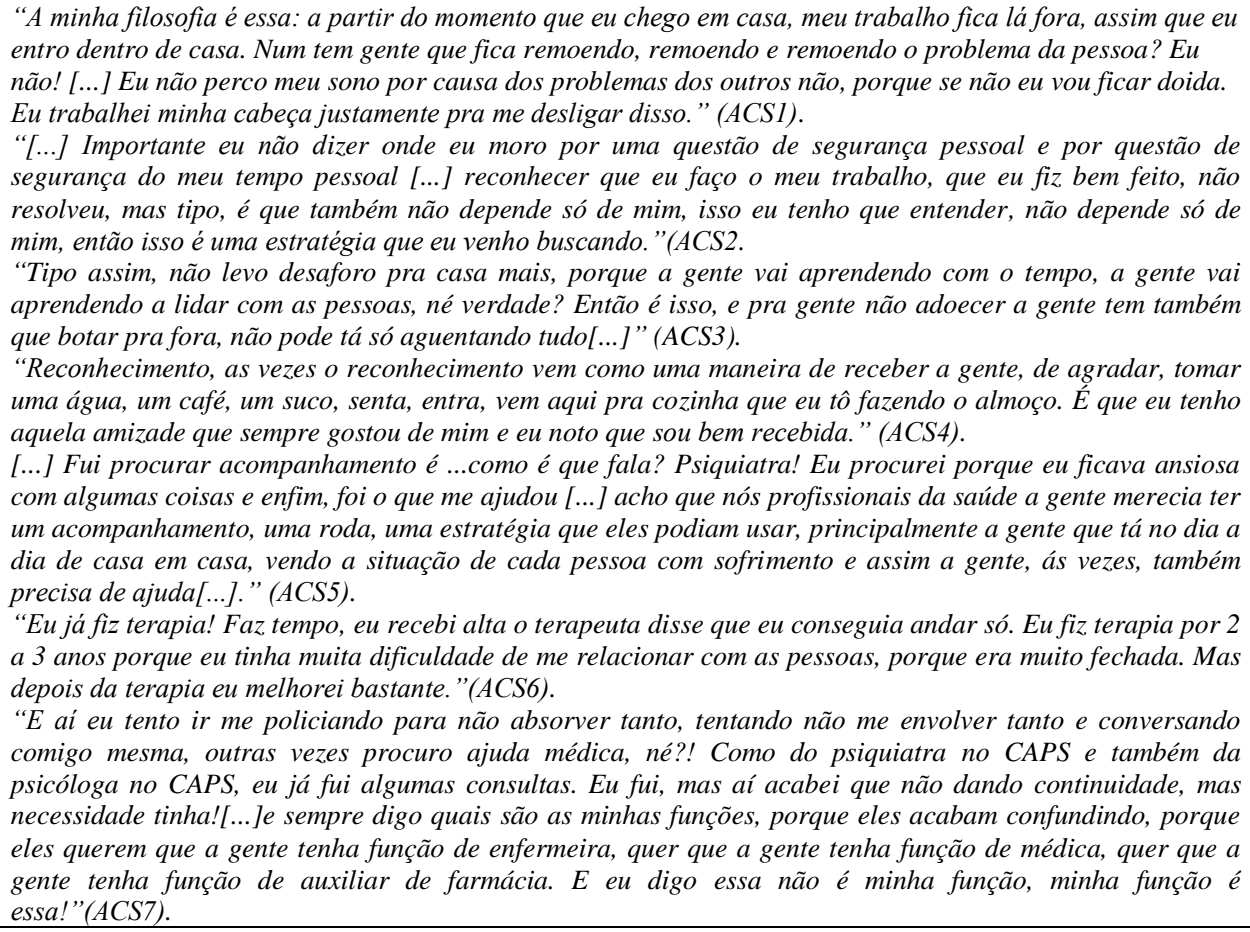 \\
\hline
\end{tabular}

Fonte: Autores (2021).

\section{Discussão}

\subsection{Interface trabalho e saúde mental}

Para que essa categoria temática seja trabalhada de forma mais abrangente é importante conceituar processo de trabalho, trabalho em saúde e saúde mental. Dessa forma, Merhy, Mishima e Santos trazem a definição de trabalho na abordagem marxista, em que o homem e todos os elementos que permitem sua (re)produção são marcados pela sua natureza histórica e social, sendo constituído por processos inter-relacionais (Santos, et al., 2018).

No que se refere ao trabalho em saúde, este possui finalidades que respondem a um projeto da sociedade em que estão presentes as concepções do que é saúde-doença-cuidado, logo é compreendido como uma prática social. Já o trabalho em equipe é aquele que envolve diferentes profissionais, não apenas da saúde, que juntos compartilham o senso de pertencimento à equipe e trabalham de maneira integrada e interdependente para atender às necessidades de saúde (Peduzzi \& Agreli, 2018).

Em uma visão micro, o processo de trabalho em saúde diz respeito ao dia-a-dia do trabalhador/profissional de saúde na produção e consumo de serviços de saúde. Estes produtos têm valor de uso e valor de troca. Este, por sua vez, faz aflorar o "trabalho abstrato", no qual o produto perde o sentido de utilidade e recebe o significado de quantidade. Esta dimensão torna o trabalhador alienado do sentido do seu trabalho, do produto e da sua consciência de trabalhador (Peduzzi, et al., 2008).

Nos processos específicos do trabalho em saúde, o objeto é o homem, portanto, deve abarcar sua subjetividade, a qual está presente nas relações estabelecidas com ele próprio, com suas partes, com a totalidade do que vem a ser, e com tudo que fazem dele um ser social. O objeto do trabalho em saúde pode ser representado pela população, ou "corpo social”, dividida em classes sociais (Paim, 2017). A necessidade de um olhar atento aos elementos que compõem o processo de trabalho em saúde, em suas relações recíprocas em função de suas particularidades, consiste em um desafio do trabalho em saúde na contemporaneidade em função das constantes mudanças no mundo do trabalho.

O sistema capitalista, pautado pela necessidade de se obter o lucro, tenciona pelo aumento da produtividade, estimula a competitividade, e impõe questões atuais, que representam desafios ao processo de trabalho em saúde, como por exemplo: 
novas formas de trabalho flexível e/ou informal, a precarização dos vínculos do trabalho, o desemprego estrutural, as questões de integralidade do cuidado e autonomia dos sujeitos, que culmina na fragmentação dos vínculos trabalhador/usuário da saúde, precariza o cuidado integral à população, que é nosso objeto do trabalho em saúde, bem como promove o aumento de doenças ocupacionais e de saúde mental nos trabalhadores da saúde (Peduzzi, et al., 2008).

A saúde mental não está limitada apenas à psicopatologia ou à semiologia, tampouco restrita ao estudo e tratamento de enfermidades mentais. A rede de conhecimentos acerca do tema de saúde mental é complexa e conta não somente com a psiquiatria, mas também com a psicologia, a psicanálise, a fisiologia, a filosofia, dentre outras. A saúde mental é, portanto, multívoca e plural enquanto campo por remeter-se às condições mentais dos sujeitos e dos coletivos, as quais são de caráter extremamente complexo (Amarante, 2007).

Desse modo, a saúde mental e é objeto-modelo produzido por meio de práticas transetoriais, sendo representada por um socius saudável que abarcaria emprego e satisfação no trabalho, um cotidiano significativo, lazer, qualidade nas redes sociais, qualidade de vida e equidade, participação social, de forma alinhada a uma pauta emancipatória social e de caráter expressamente político. Deve-se pensar o conceito de saúde mental como "integrais de saúde-enfermidade-cuidado", isso porque esse objeto modelo só é definido em sua conformação inteira, visto que é composto por facetas, ângulos encobertos, regiões sombreadas, e que cada vez que se olha para cada uma dessas regiões e ângulos não acessamos de fato o conjunto das propriedades que definem o objeto modelo "saúde mental"(Filho, et al., 1999).

No que concerne a relação dos processos de trabalho com a saúde mental das ACS, estas afirmaram que tem influência sim, devido as pressões e cobranças, grande quantidade de famílias sobre sua responsabilidade, muitos impasses na microárea e acúmulo de trabalho. Estudo realizado por Andrade et al corrobora com esta pesquisa, pois afirma que o trabalho do agente comunitário de saúde expõe estes profissionais a situações de cansaço, ansiedade, estresse, distúrbios gastrointestinais e respiratórios (Andrade, et al., 2018).

Ademais, pesquisas demonstram que situações como desvalorização do trabalho, pressão por parte de usuários, equipe e gestores, assim como a não resolutividade dos problemas da população pelo sistema de saúde acaba sobrecarregando o ACS, pois essas necessidades perpassam a sua governabilidade causando sofrimento pelo conflito entre o que é idealizado e a expectativa em relação a sua competência (Almeida et al., 2016; Araujo et al., 2018).

Além disso, as participantes desse estudo são todas mulheres e esse trabalho excessivo está relacionado a dupla ou tripla jornada, pois além da carga horária de trabalho formal, realizam atividades voltadas ao cuidado da casa, família e filhos com turnos extras (Moreira et al., 2016 ; Fernandes et al., 2018).

Também foi citado pelas ACS como dificuldade geradora de estresse e ansiedade o contexto de trabalho na pandemia causada pelo coronavírus, devido a disponibilização do número do celular para os usuários com o objetivo de garantir a continuidade do cuidado as famílias. As agentes comunitárias de saúde, como foi observado nas falas, que isso causou diminuição da privacidade e maior tempo de trabalho, em razão das pessoas não respeitarem o horário de trabalho. Estudo de Menegussi et al. evidenciou que as atividades laborais realizadas pelos ACS são complexas e permeadas pelo desgaste emocional em comparação as demais classes profissionais do SUS, por ser trabalhador da saúde no local onde vive (Menegussi, et al., 2014).

Segundo Oliveira as condições de trabalho dos agentes comunitários interferem tanto na saúde como no modo de pensar, agir e sentir desses trabalhadores, ocasionando agravos psicológicos, estresse e sobrecarga psíquica. Para mais, estão expostos nos territórios que atuam a situações de violências, assaltos, estupros, tráfico de drogas, prostituição, enfermidade, miséria e morte, o que poderá levar a despertar sentimentos como frustração e impotência (Oliveira \& Nery, 2019).

É notório que o contexto de trabalho que as pessoas vivenciam tende a ignorar o tema sofrimento psíquico, porém dar visibilidade a esse sofrimento é entender uma das grandes consequências da insistida vivência do sujeito em um ambiente que 
não lhe é favorável (Oliveira, 2019). Para Dejours a forma de organização do trabalho executa uma ação específica no homem que pode afetar seu estado psicológico. Diante de algumas condições pode surgir um sofrimento, o qual pode advir de choques entre história individual que guarda projetos, expectativas, desejos diante de uma instituição laboral que os ignora (Dejours, 1987).

A psicodinâmica do trabalho realiza estudos sobre o adoecimento e sofrimento psíquico relacionado ao ambiente de trabalho, a dinâmica das organizações do trabalho e a subjetividade dos trabalhadores. Investigando a organização do contexto do trabalho, as condições e relações de trabalho, as vivências de prazer e sofrimento do trabalhador e como se configura suas estratégias defensivas (Oliveira, 2019).

O sofrimento mental pode estar presente na vida do trabalhador a partir do momento que ele não é capaz de desempenhar o seu trabalho de forma adequada, não cumprindo as determinações. Associado a isso, tem-se a histórica vivência do homem dentro das organizações, onde o trabalho era relacionado a divindade, a escravidão, tendo um caráter humilhante e degradante, repercutindo para um processo de esquecimento de identidade e provocando um crescimento de patologias vinculadas ao exercício de função (Guanaes-Lorenzi \& Pinheiro, 2016).

O sentimento de impotência diante das demandas do território e a falta de resolutividade do sistema de saúde foram bastante frequentes nas falas das participantes. Esse sentimento também foi relatado em outras pesquisas, onde o agente comunitário de saúde não consegue abarcar todas as exigências da comunidade, pois determinadas situações requerem capacidade e criatividade que ultrapassam seu poder de ação. Associado a isso, a população e o próprio Agente Comunitário de Saúde não conhecem qual o seu papel profissional, fazendo com que o mesmo assuma para si atividades que competem a outros integrantes da equipe, como funções administrativas e agendamento de consultas (Santos, et al., 2018)

Esse mesmo estudo designou um conjunto de elementos relacionados à carga física no contexto socioambiental de trabalho do Agente Comunitário de Saúde, tais como a exposição física na comunidade, estrutura precária na unidade de saúde e risco de violência por parte da comunidade. No que se refere ao contexto de violência no território, as falas anteriormente citadas pelas ACS entrevistadas indicam situações de agressão verbal, em que as ACS são as primeiras a serem responsabilizadas e culpadas pela comunidade, além disso as falas indicam sentimento de frustração diante dos casos de violência contra a criança, mulher e idoso (Lopes, et al., 2018).

Diante desse cenário, é importante a criação de espaços de escuta acolhedora para compartilhamento das experiências e vivências dos Agentes Comunitários de Saúde, a fim de oportunizar a reflexão sobre as demandas encontradas no cotidiano de trabalho e trazer resolutividade através do trabalho em equipe, evitando agravos ocupacionais (Santos, et al., 2018).

Os serviços e tarefas realizadas pelos ACS não devem ultrapassar os seus limites, de modo a fortalecer o alcance de suas metas. Ademais, é necessário o provimento de tempo para abordagem dos casos, mecanismos de formação continuada e ampliação dos espaços de falas sobre os estressores vivenciados no cotidiano de trabalho (Alcântara \& Assunção, 2016).

Christophe Dejours aponta que o sofrimento psíquico no ambiente de trabalho evidencia uma disputa, na qual por um lado há procura pelo bem-estar e, por outro, há chance de comprometimento da saúde mental. Ressalta que não há uma normalidade psíquica no trabalho, pois os trabalhadores frequentemente são expostos nesse ambiente a situações que os afetam, que os constrangem. $\mathrm{O}$ autor estudou com profundidade a psicodinâmica do trabalho, e tentou analisar quais estratégias eram adotadas pelo trabalhador para possibilitar a superação do sofrimento psíquico pelo prazer no cotidiano do seu trabalho (Dejours, 1993).

\subsection{Estratégias de cuidado em saúde mental}

É importante a reflexão sobre o significado da palavra cuidado, pensada não apenas como um conjunto de medidas terapêuticas e procedimentos destinados a tratar e/ou curar um corpo biológico, mas numa dimensão ampliada em que deve ser 
considerada a existência da pessoa no mundo, o ambiente em que ela vive, os estressores do cotidiano que está exposta e as relações intrapessoais e interpessoais. Cuidado é arte de produzir potência no outro, independentemente das circunstâncias (Franco \& Hubner, 2019).

As estratégias de cuidado em saúde mental relatadas pelas ACS foram diversificadas o que remete ao entendimento diferente do que é cuidado para cada uma. A fala da entrevistada 5 foi bem marcante no que se refere a importância da realização de rodas, de momentos que possibilitem conversas e trocas relacionadas ao trabalho, assim como foi relatada a necessidade de terem um acompanhamento psicológico por vivenciarem de perto o sofrimento das pessoas, reconhecendo que elas também precisam de ajuda.

Pesquisa realizada com nove profissionais que atuavam na Atenção Básica, em um Centro de Atenção Psicossocial (CAPS) e em Centros de Referência em Saúde do Trabalhador (CEREST) do estado de São Paulo, evidenciou que a realização de grupos terapêuticos evita o isolamento, proporciona o compartilhamento de experiências e potencializa o enfrentamento de dificuldades encontradas na realidade social e de trabalho, possibilitando que os profissionais de saúde percebam como seus problemas se relacionam com o trabalho (Souza \& Bernardo, 2019).

Foi apontado pelas entrevistadas como estratégia de autocuidado a tentativa de instituir limites entre a vida social na comunidade e a vida profissional. A dupla inserção (institucional e comunitária) deste trabalhador gera interfaces diferentes na relação com as pessoas, pois ora a convivência no território favorece o vínculo e o repasse de informações, ora os ACS não possuem controle do seu tempo de trabalho, devido os usuários se aproveitarem dos espaços de convivência na comunidade para cobrarem demandas referentes ao trabalho (Chuengue \& Franco, 2018).

Os recursos individuais são estratégias que os trabalhadores utilizam, muitas vezes de maneira inconsciente, para opor-se ao próprio sofrimento e ao sofrimento do outro no trabalho, ou racionalizar, evitar a angústia, sofrimento, medo e insegurança vividos no trabalho (Dejours, 1994).

É de grande relevância destacar que o cuidado de si incorporado pelos Agentes Comunitários de Saúde perpassa a sua relação com o outro. Segundo Foucault (2004):

O cuidado de si traz essa dimensão ética que pode ser entendida também como o cuidado do outro, uma vez que o cuidado de si implica uma relação com o outro. Contudo, "não se deve fazer passar o cuidado dos outros na frente do cuidado de si; o cuidado de si vem eticamente em primeiro lugar na medida em que a relação consigo mesmo é ontologicamente primária.

O reconhecimento pela comunidade do trabalho dos ACS e as amizades construídas foram citadas como estratégias de cuidado, pois em pequenos gestos, como o de ser bem recebido durante a visita domiciliar, o profissional se sente cuidado e valorizado. Bezerra e Feitosa demonstraram em sua pesquisa que os agentes comunitários de saúde expressaram sentimentos de amor, amizade e vínculo com as famílias, sendo estes potencializadores das ações dos ACS, fazendo emergir uma implicação positiva com o território, mesmo diante das dificuldades (Bezerra \& Feitosa, 2018).

Outras iniciativas favoráveis à saúde mental constatadas foram: não dizer o endereço da sua residência para os usuários, não se envolver exageradamente com os problemas do território, explicar qual a sua função enquanto ACS para que não seja cobrado algo além da sua governabilidade. Dessa forma, há uma busca de ressignificação do sofrimento na medida que as agentes de saúde constroem estratégias de resistência às situações do cotidiano de trabalho (Cremonese, et al. 2013).

\section{Considerações Finais}

Pode-se inferir que o artigo buscou compreender os fatores que ocasionavam sofrimento mental na rotina laboral dos Agentes Comunitários de Saúde, mas sua análise se deu de forma mais geral, limitando-se a identificar tais fatores sem 
aprofundá-los, o que desdobra-se numa recomendação à outros estudos, os quais possam se debruçar em análises profundas de temas aqui evidenciados, como por exemplo, a questão de gênero, visto que a maioria das ACS são mulheres, e o que é ser mulher em suas particularidades, trabalhadora do SUS e moradora desse território de atuação ao mesmo tempo? Recomendase analisar as violências a que estão expostas no cotidiano dos serviços. E, também, que futuras pesquisas possam debruçar-se sobre o desafio no âmbito público-privado que tais trabalhadoras enfrentam com sua privacidade, a qual muitas vezes se mostra invadida pela demanda da própria comunidade.

Este estudo também apontou as estratégias de cuidado citadas pelas agentes comunitárias de saúde para enfrentamento do contexto adoecedor no ambiente de trabalho em que estão inseridos, tais como instituir limites entre a vida pessoal e a profissional, orientar os usuários sobre o seu papel enquanto agente comunitária de saúde, não se envolver com os problemas do território e reconhecer suas limitações enquanto ACS. Também foi mencionado pelas participantes a importância da promoção de espaços de fala e escuta, em que a equipe de saúde pudesse dialogar sobre os desafios e prazeres vivenciados no trabalho.

Dessa forma, é fundamental o planejamento de ações e estratégias para minimizar os processos adoecedores relacionados ao processo de trabalho do agente comunitário de saúde, com o propósito de melhorar as condições de trabalho, promover saúde mental, contribuir na qualidade de vida e, consequentemente, melhorar o cuidado prestado à população.

A realização do estudo possibilitou a identificação de características peculiares à realidade de um único cenário, devido a pesquisa ter incluído apenas ACS de uma única UBS da zona urbana. Além disso, foi evidenciado aspectos psicossociais vividos apenas por estes profissionais, isso suscita a necessidade de outras pesquisas que possam explorar o tema envolvendo os demais profissionais da saúde, revelando perspectivas não contempladas pelo estudo.

\section{Referências}

Alcântara, M. A. de, \& Assunção, A. Á. (2016). Influência da organização do trabalho sobre a prevalência de transtornos mentais comuns dos agentes comunitários de saúde de Belo Horizonte. Revista Brasileira de Saúde Ocupacional, 41(0), 1-11.

Almeida, M. C. dos S., Baptista, P. C. P., \& Silva, A. (2016). Cargas de trabalho e processo de desgaste em Agentes Comunitários de Saúde. Revista Da Escola de Enfermagem, 50(1), 95-103.

Amarante, P. (2007). Saúde mental, territórios e fronteiras. Fiocruz.

Andrade, C. C. B., Paschoalin, H. C., Sousa, A. I., Greco, R. M., \& Almeida, G. B. S. (2018). Agentes comunitários de saúde: perfil sociodemográfico, condições laborais e hábitos de vida. Revista de Enfermagem UFPE on Line, 12(6), 1648.

Araujo, E. F. dos S., Paz, E. P. A., Ghelman, L. G., Mauro, M. Y. C., Donato, M., \& Farias, S. N. P. de. (2018). Os agentes comunitários de saúde nas práticas educativas: potencialidades e fragilidades. Revista Enfermagem UERJ, 26, e18425.

Bezerra, Y. R. do N., \& Feitosa, M. Z. de S. (2018). A afetividade do agente comunitário de saúde no território: Um estudo com os mapas afetivos. Ciencia e Saude Coletiva, 23(3), 813-822.

Brasil (2016) Conselho Nacional de Saúde. Resolução nº 510, de 07 de abril de 2016. Diário Oficial da União.

Brasil (2012) Conselho Nacional de Saúde. Resolução nº 466, de 12 de dezembro de 2012.Aprova as diretrizes e normas regulamentadoras de pesquisas envolvendo seres humanos. Diário Oficial da União.

Brasil (2002) Lei n ${ }^{\circ}$ 10.507, de julho de 2002. Diário Oficial da União.

Brasil (2006) Lei no 11.350 de 05 de outubro de 2006. Diário Oficial da União.

Chuengue, A. P. G., \& Franco, T. B. (2018). O reconhecer e o lidar dos agentes comunitários de saúde diante da bioética: entre a ética do cuidado e os poderes disciplinares. Physis: Revista de Saúde Coletiva, 28(4), 1-18.

Cremonese, G. R, Motta, R. F, \& Traesel, E. (2013). Implicações do trabalho na saúde mental dos Agentes Comunitários de Saúde. Cadernos de Psicologia Social Do Trabalho, 16(2), 279-293.

Dejours, C. (1987). A loucura do trabalho: estudo de psicopatologia do trabalho. Cortez Editora, (5a ed.), 1-168.

Dejours, C. (1994). Psicodinâmica do trabalho: contribuições da escola dejouriana à análise da relação prazer, sofrimento e trabalho. Atlas.

Dejours, C. (1993). Uma nova visão do sofrimento humano nas organizações. O indivíduo na organização: dimensões esquecidas. Atlas. 
Fernandes, G. A. B., Coelho, A. da C. O., Paschoalin, H. C., Sarquis, L. M. M., \& Greco, R. M. (2018). Demandas Psicológicas, Controle E Apoio Social No Trabalho De Agentes Comunitários De Saúde. Cogitare Enfermagem, 23(4).

Filho, N. D. A., Coelho, M. T. Á., \& Peres, M. F. T. (1999). O conceito de saúde mental. Revista USP, O(43), 100.

Foucault, M. (2004). Ética, sexualidade e política (Ditos e Escritos, V). Forense Universitária.

Franco, T. B., \& Hubner, L. C. M. (2019). Clínica, cuidado e subjetividade: afinal, de que cuidado estamos falando? Saúde Em Debate, 43(spe6), 93-103.

Guanaes-Lorenzi, C., \& Pinheiro, R. L. (2016). A (des)valorizeção do agente comunitário de saúde na Estratégia Saúde da Família. Ciencia e Saude Coletiva, $21(8), 2537-2546$.

Lopes, D.M.Q, Filho, W.D.L, Beck, C.L.C, Coelho, A. P. . (2018). Cargas De Trabalho Do Agente Comunitário De Saúde: Pesquisa E Assistência Na Perspectiva Convergente-Assistencial. Texto \& Contexto - Enfermagem, 27(4), 1-10.

Menegussi, J.M, Ogata, M.N, Rosalini, M. H. . (2014). O Agente Comunitário De Saúde Como Morador, Trabalhador E Usuário Em São Carlos,São Paulo. Trab. Educ. Saúde, Rio de Janeiro, 12(1), 87-106.

Moreira, I. J. B., Horta, J. A., Duro, L. N., Borges, D. T., Cristofari, A. B., Chaves, J., Bassani, D. C. H., Cerizolli, E. D., \& Teixeira, R. M. (2016). Perfil sociodemográfico, ocupacional e avaliação das condições de saúde mental dos trabalhadores da Estratégia Saúde da Família em um município do Rio Grande do Sul, RS. Revista Brasileira de Medicina de Família e Comunidade, 11(38), 1-12.

Oliveira, J. D. S., \& Nery, A. A. (2019). Condições de trabalho e saúde de agentes comunitários de saúde. Revista de Enfermagem UFPE on Line, $13(5), 1503$.

Oliveira, L. A. (2019). O prazer-sofrimento psíquico no trabalho e a perspectiva de Christophe Dejours. Revista Psicologia \& Saberes, 8(11), 360-369.

Paim, J. S., (2017). Sociedade, saúde e história. Porto Alegre: Rede Unida.

Peduzzi, M., \& Agreli, H. F. (2018). Teamwork and collaborative practice in primary health care. Interface: Communication, Health, Education, 22, 15251534.

Peduzzi, M., Schraiber, B., Do, N., Pioneiramente, C., Cec, M., \& Donnangelo, F. (2008). Marina Peduzzi e Lília Blima Schraiber. 1-11.

Pereira, A. M., Jucá, A. L., Lima, I. de A., Facundes, V. L. D., \& Falcão, I. V. (2018). A qualidade de vida do agente comunitário de saúde e possíveis contribuições da terapia ocupacional. Cadernos Brasileiros de Terapia Ocupacional, 26(4), 784-796.

Santos, A. C, Hoppe, A. S, \& Krug, S. B. (2018). Agente Comunitário de Saúde: implicações dos custos humanos laborais na saúde do trabalhador. Physis: Revista de Saúde Coletiva, 28(4), 1-18.

Santos, D. de S., Mishima, S. M., \& Merhy, E. E. (2018). Processo de trabalho na Estratégia de Saúde da Família: Potencialidades da subjetividade do cuidado para reconfiguração do modelo de atenção. Ciencia e Saude Coletiva, 23(3), 861-870.

Silva Filho, O. C. da. Minayo M. C. S., \& Costa A. P. (2019). Técnicas que fazem uso da Palavra, do Olhar e da Empatia: Pesquisa Qualitativa em Ação. Aveiro: Ludomedia, 2019. Ciência \& Saúde Coletiva, 25(5), 1991-1992.

Souza, H. A., \& Bernardo, M. H. (2019). Prevenção de adoecimento mental relacionado ao trabalho: a práxis de profissionais do Sistema Único de Saúde comprometidos com a saúde do trabalhador. Revista Brasileira de Saúde Ocupacional, 44, 1-8.

Speroni, K. S, Fruet, I. M. A, Dalmolin, G. L, \& Lima, S. B. (2016). Percepções dos agentes comunitários de saúde: contribuições para a gestão em saúde. Revista Cuidarte, 7(2), 1325-1337. 